\title{
Lettres inédites de Pierre Loti à la princesse Mathilde Bonaparte et au comte Joseph Primoli
}

\section{Anna Maria Scaiola}

\section{(2) OpenEdition}

\section{Journals}

\section{Édition électronique}

URL : http://journals.openedition.org/rief/1232

DOI : 10.4000/rief.1232

ISSN : 2240-7456

\section{Éditeur}

Seminario di filologia francese

\section{Référence électronique}

Anna Maria Scaiola, « Lettres inédites de Pierre Loti à la princesse Mathilde Bonaparte et au comte Joseph Primoli », Revue italienne d'études françaises [En ligne], 6 | 2016, mis en ligne le 15 décembre 2016, consulté le 30 avril 2019. URL : http://journals.openedition.org/rief/1232 ; DOI : 10.4000/ rief.1232

Ce document a été généré automatiquement le 30 avril 2019.

\section{$\oplus \Theta \Theta$}

Les contenus de la RIEF sont mis à disposition selon les termes de la Licence Creative Commons Attribution - Pas d'Utilisation Commerciale - Pas de Modification 4.0 International. 


\title{
Lettres inédites de Pierre Loti à la princesse Mathilde Bonaparte et au comte Joseph Primoli
}

\author{
Anna Maria Scaiola
}

\section{à la mémoire de Massimo Colesanti}

La Bibliothèque de la Fondation Primoli de Rome, relancée et dirigée par son Président, Massimo Colesanti, possède environ 50.000 volumes et un précieux patrimoine de documents, de manuscrits, d'autographes, de photographies et de lettres, encore en partie inédits, ainsi qu'une riche section consacrée à Stendhal comprenant des textes rares et annotés et des essais critiques. Le comte Giuseppe, ou Joseph, Primoli, arrièrepetit-fils aussi bien de Lucien Bonaparte que de Joseph Bonaparte, était un collectionneur d'art et un bibliophile, ainsi qu'un photographe de talent, animateur et habitué des salons romains et parisiens. Ce singulier personnage, à la fois mondain, lettré et écrivain, qui se partageait entre l'Italie et la France, a annoté en français, pendant des années, sur des cahiers et des carnets, selon un rythme discontinu, des épisodes et des fragments de vie quotidienne, des notes intimes, des souvenirs, des portraits de famille, des témoignages et les comptes rendus de ses rencontres avec des hommes de lettres de son temps... Dans ses «mémoires" autographes, le comte raconte les étés qu'il a passés, quand il était adolescent, dans la demeure de Saint-Gratien, la résidence d'été de sa tante, la princesse Mathilde Bonaparte, qui transplantait son brillant salon parisien dans le Val-d'Oise dès les premières chaleurs :

Je passais donc mes vacances à $S^{t}$ Gratien, chez ma tante Mathilde, où j'avais l'heureuse fortune de voir défiler et d'entendre causer des artistes et des savants, des poètes et de grands écrivains, des hommes distingués dans tous les genres : Mrs. Sainte-Beuve, Taine, Flaubert, les de Goncourt, Augier, Gautier, Alfred Arago, Lavoix, Marchal, Baudry, Boulanger, Carpeaux, Anastasi, Giraud, Hébert, Cabanel, etc. Mettez comme d'union entre ces talents si divers la Châtelaine qui sait à fond l'Art de donner le ton à la causerie, d'en fournir le thème et d'en varier les motifs à l'infini. Incomparable chef d'orchestre qui met d'accord tous les musiciens entre 
eux, dirige la conversation depuis l'ouverture et ne la laisse pas tomber avant le bonsoir final. ${ }^{1}$

2 Les documents concernant Loti, qui sont conservés à la Fondation Primoli, permettent d'ajouter un certain nombre d'éléments peu connus ou inédits à sa biographie. En particulier, ses lettres autographes à la princesse Mathilde et au comte Primoli confirment ses contacts avec les descendants italiens des Bonaparte, tout en levant un doute - avec l'appui d'Alain Quella-Villéger, le plus grand spécialiste actuel de Loti quant à une visite hypothétique de l'écrivain-voyageur à Rome: une destination non désirée et manquée, bien que des sources italiennes attestent la présence de Loti dans la capitale $^{2}$.

Loti n'a eu en effet que peu de contacts avec l'Italie et sa réception y a été incertaine. La première monographie italienne de Loti «homme de lettres» est celle d'Americo Bertuccioli, professeur de langue française de la « Reale Accademia Navale » de Livourne, qui écrit en 1926 : « Il Loti non aveva mai nutrito, chissà perché ?...(Vieti pregiudizi ?... Errata valutazione di certe condizioni politiche ?...) una gran simpatia per la giovine Italia $»^{3}$. Bertuccioli est aussi le traducteur d'Impressions d'Italie $e^{4}$, où Loti aurait exprimé, selon lui, toute son admiration pour la valeur des soldats italiens confrontés à des conditions difficiles sur le front, à l'occasion de sa visite sur le Carso, en août 1917. En revanche, Ugo Ojetti, qui fut chargé d'accompagner cet hôte illustre dans sa visite d'Aquilée et de Grado, trace de lui un portrait ironique : ce vieillard «languido e timido», au teint terreux de malade ravivé - selon le cliché - par une couche de poudre, est fatigué, haletant, préoccupé que «Danonziò » ait reçu un de ses livres. Loti lui paraît s'ennuyer et se désintéresser de ce qui l'entoure et, ajoute Ojetti, dépourvu de rhétorique guerrière : «Oserei dire che nemmeno la guerra italiana molto gli importava ${ }^{5}$.

4 La Bibliothèque de la Fondation Primoli possède plus de trente livres de Loti, publiés par Calman Lévy ${ }^{6}$, dont certains avec l'indication de leur propriétaire, comme Le Roman d'un enfant (1890) : "Joseph Primoli, Roma 26 mai 1890 ». Le comte appréciait les récits de voyage de Loti pour son talent de "peintre » évocateur et visionnaire, puisqu'il écrit en mars 1899 ces lignes - inédites - dans le manuscrit autographe de ses mémoires, conservé à la Fondation :

Je ne me sens pas attiré par les récits de voyage : à moins que ces livres ne soient signés par Pierre Loti qui a le pouvoir de transposer sur le papier le tableau, les couleurs, l'air ambiant, l'atmosphère. Ces photos, plus au moins au point, ces fleurs desséchées, ces guides démasqués m'ennuient mortellement. ${ }^{7}$

Sur la première page des Désenchantées, roman des harems turcs contemporains (1906), le comte a annoté : « Lu entre Patras et Chalchis - en allant à Constantinople sur le Nirvana, 4-5 août 1906, Joseph Primoli ». Et au-dessous, avec un trait de crayon plus léger : "Relu du 14 au 16 août en vue de Beicos ». Henri de Régnier participe à ce voyage, auquel il consacre un compte rendu ${ }^{8}$.

6 Sur un exemplaire d'Un Pèlerin d'Angkor (1912), la dédicace est de Loti lui-même : "Au Comte Joseph Primoli, en souvenir de nos déjeuners de naguère en commun à SaintGratien ». L'évocation en 1912 de cette convivialité au château de Saint-Gratien dénote une habitude de fréquentation dans le passé avec la princesse et le comte, qui se poursuit même après la disparition de la noble dame en 1904.

7 À la demande du comte, Loti avait participé au fascicule grand format Roma: raccolta artistico-letteraria internazionale publié en 1897 à l'initiative de la princesse Pallavicini ${ }^{9}$ avec l'aide d'un groupes de dames de la haute société romaine. Deux cent vingt artistes, 
musiciens et gens de lettres avaient répondu à l'appel du «Comité Charité et Travail $»^{10}$. Le comte Primoli avait lancé un appel à contribution à ses correspondants français, qui répondirent avec des textes courts, dont certains furent reproduits manuscrits : Régnier, Coppée, Gregh, France, Barrès, les frères Daudet, Prévost, Bourget, Zola et même Mallarmé ${ }^{11}$, mais aussi Pascoli et D'Annunzio, et Loti, qui envoie ces lignes - insérées à la p. 21 des quarante-six que comprend le recueil - de la part des pauvres, des simples, qui possèdent des valeurs et des qualités, comme une gentillesse naturelle, dont les classes supérieures et plus cultivées sont dépourvues :

Pour ne pas blesser les petits, pour ne pas faire de peine - surtout à ceux qui souffrent - il y a un certain tact exquis et rare, qui vient du cœur, que les plus humbles possèdent souvent par nature, mais qu'en revanche l'éducation ne donne jamais, même aux gens les plus affinés du monde, Pierre Loti

8 Le Musée Napoléonien de Rome, installé au premier étage du palais Primoli, via Zanardelli, conserve le billet autographe $\mathrm{n}^{\circ} 6108$ de Loti avec cette phrase édifiante, qui n'est pas sans rappeler le ton évangélique du « Rapport sur le Prix de vertu » lu au cours de la séance publique annuelle de l'Académie Française du 17 novembre 1898, et repris dans Le Château de la Belle au bois dormant (1910) sous le titre significatif de «Ceux devant qui il faudrait plier le genou ». Ce billet est bordé de noir en signe de deuil, probablement pour la mort de la mère de Loti, survenue en novembre 1896, mort dont son fils fait dans son Journal ${ }^{12}$ un long récit empreint d'une émotion qui témoigne d'un poignant sentiment d'abandon et de mélancolie, d'une angoisse du vide et de "funèbres apparitions crépusculaires $»^{13}$ : la mère disparue est évoquée douloureusement et fréquemment, regrettée avec nostalgie, fantasmée et rêvée pendant des nuits et des nuits, souvent de suite.

Le billet autographe $n^{\circ} 1880$ conservé par la Fondation, également bordé de noir, envoyé en retard au comte pour la publication, se réfère à cette réflexion :

Monsieur,

Je suis désolé du retard involontaire que j'ai mis à vous envoyer la petite phrase demandée, et je tremble qu'elle n'arrive trop tard!

Veuillez, je vous en prie, me pardonner, monsieur, et agréer encore l'expression de mon plus sympathique dévouement

Pierre Loti

10 La Bibliothèque de la Fondation Primoli possède aussi un exemplaire des Reflets sur la sombre route de novembre 1899, avec une dédicace de Loti: "Pour mon ami Lucien Jousselin ». Lucien-Hervé Jousselin ${ }^{14}$, officier de marine, s'était occupé du contrat d' Aziyadé (dans lequel il apparaît sous le nom de Plumkett) avec Calmann Lévy, il avait été le réviseur des premiers manuscrits de Loti et le cosignataire des Fleurs d'ennui de 1883 ( $«$ en collaboration avec $\mathrm{H}$. Plumkett »). Le Musée Napoléonien possède des lettres autographes inédites de Jousselin à Giulia del Gallo di Roccagiovine, la tante du comte Primoli ${ }^{15}$. Une lettre de Jousselin, datée de 1897 par Giulia, postérieure au mois de mai de cette année en raison de références internes, précise :

Le petit paquet ci-joint renferme plusieurs livres de Loti, qui vous intéresseront sans doute. Certains sont en bien mauvais état, mais je les ai beaucoup prêtés et certaines personnes sont plus soigneuses du bien que d'autres.

11 Jousselin avait été attaché naval à Rome entre 1896 et 1898, puis, entre 1899 et 1900, capitaine de Frégate, toujours à Rome. Deux lettres ${ }^{16}$ de Loti à Jousselin sont apparues lors d'une vente aux enchères qui s'est tenue à Avignon (Hôtel des ventes; fonds Fernand 
Laplaud) le 21 janvier 2016. L'écrivain refuse les invitations à Rome que lui a adressées son correspondant: la première lettre ( $\left.{ }^{\circ} 279\right)$ est postée d'Hendaye (cachet illisible), dans son Pays Basque bien aimé, où Loti achètera en août 1903 une maison. La deuxième lettre de Loti datée "Hendaye, 20 mars» commence sur le ton de l'impatience: "Comment voulez-vous que j'aille vous voir à Rome quand je commande ici le Javelot à la frontière d'Espagne?». Loti reprend le commandement du Javelot du 20 mai 1896 à la fin octobre 1897 : le 20 mars de la deuxième lettre pourrait donc se référer à l'année 1897. Selon son Journal, le vendredi 19 mars, Loti est à Hendaye, qu'il décrit, comme dans d'autres passages, en exaltant sa chaleur accueillante, comparée à la maison de sa ville natale de Rochefort, vide et froide à cause de l'absence de sa mère, dont le souvenir est constamment lié à la tiédeur du soleil et surtout aux fleurs, qui reviennent avec une grande variété également dans ses écrits intimes :

Arrivé de Rochefort, après deux jours passés dans la triste maison, au printemps incertain ; - déjà, dans la cour, était fleuri le dielytra rose, que maman ne verra jamais plus...

Et ici, j'ai trouvé le printemps plus chaud, la griserie du soleil - Dans le vieux jardin abandonné de la maison voisine, boutonnent les pivoines roses, qui me rappellent des souvenirs d'un printemps passé depuis déjà six années... ${ }^{17}$

Plusieurs lettres encore inédites ${ }^{18}$ conservées à la Fondation Primoli concernent elles aussi des invitations réitérées à Rome du comte à Loti, lequel, malgré la médiation de la princesse Mathilde, ne donne pas suite, en dépit de quelques hésitations et de quelques promesses. Le comte recevra au Palais Primoli des intellectuels et des artistes français comme Bourget, France, Maupassant, Debussy... Mais Alain Quella-Villéger, que j'ai interrogé, m'a répondu à ce sujet, de manière définitive, et je l'en remercie beaucoup, le 18 novembre 2015 :

Je crois pouvoir certifier que Pierre Loti n'est jamais allé à Rome et n'en a jamais eu le désir. Il s'est contenté des escales maritimes, comme à Naples, ou bien de se rendre à Venise (notamment pour y rencontrer la reine Élisabeth de Roumanie en exil) ${ }^{19}$. Il faudrait que je fasse quelques recherches pour voir si je trouve d'autres mentions au sujet du comte Primoli ou de la princesse Mathilde.

Nous transcrivons ces lettres inédites de Loti, en essayant de reconstruire leur séquence, ce qui n'est pas évident car elles sont généralement sans date ni ordre chronologique, avec une petite numérotation manuscrite, et conservées dans deux classeurs différents : le premier, catalogué «scat. XXXI, n. 12360-12363", contient les lettres à la princesse Mathilde, et le deuxième les lettres au comte : «scat. II, n. 1874-1883 ». Ces lettres, écrites entre la fin du XIX et le début du XXe siècle, puis entre la fin de l'année 1903 et le début de 1904, témoignent d'un rapport formel durable, empreint de courtoisie et de respect, sans pour autant négliger les marques d'amitié et les déclarations de sympathie.

Loti est en contact avec la princesse Mathilde à Paris, comme l'atteste son Journal à la date du jeudi 2 juin 1892 : « Vote à l'Académie. L'après-midi chez la princesse Alice, - où vient la vieille princesse Mathilde prendre le thé de 5 heures $»^{20}$. Le 8 juin 1893, Loti est à Paris chez la princesse Alice de Monaco qui est malade, et il écrit dans son Journal: «[...] - et elle continuera tant qu'elle en aura encore la force sa vie de petite souveraine élégante : ce soir, malgré la fièvre qui lui met un feu rose aux joues, elle ira chez la princesse Mathilde $»^{21}$.

Un groupe de lettres inédites conservées à la Fondation Primoli se présente sur du beau papier, certaines avec l'élégant monogramme des initiales du pseudonyme de l'écrivain $P L$ et de celles de son vrai nom JV - Julien Viaud, dans le coin en haut à gauche, orné d'un 
cartouche en exergue dans lequel est écrit : « Mon mal j'enchante », la devise bien connue de $\operatorname{Loti}^{22}$.

Le deux premières lettres, la lettre $n^{\circ} 12360$ à la princesse et la $n^{\circ} 1874$ au comte, sembleraient être isolées par rapport à celles qui suivent, en succession et croisées.

1) La lettre $n^{\circ} 12360$ à la princesse Mathilde, sur papier avec monogramme et cartouche, date probablement de décembre 1894, car Loti demande à être reçu le samedi, qui tombait précisément le 1er décembre cette année-là, pour fixer un rendez-vous. Selon son Journal, Loti quitte Hendaye pour Paris le 28 novembre et il y reste jusqu'au 6 décembre. Pendant son séjour, il rend visite à la princesse J. Ghyka et à Laetitia de Savoie, et il rejoint Alice de Monaco au château de Marchais (Aisne) ${ }^{23}$ :

Princesse amie,

Voudrez-vous être assez bonne pour m'envoyer tout de suite un petit mot disant si vous voulez de moi le samedi 1er Décbre, - et, pour le cas où ce jour me serait impossible, si je pourrais aussi bien me présenter le 2, le 3 ou le 4.

Respectueusement à vous de tout cœur,

P. Loti

2) La lettre $n^{\circ} 1874$ au comte Primoli, sur papier à lettre uniquement avec un cartouche et la devise, a une datation incertaine : été 1894 ? L'indication concernant le retour d'Orient pourrait se référer au voyage que Loti a effectué du début février au début juin 1894. Loti trouve dans le courrier qui s'est accumulé pendant ses mois d'absence une vieille lettre du comte, qui joue le rôle d'intermédiaire pour le compte de "Sa Majesté », probablement l'Impératrice Eugénie, dont Primoli était très proche. Loti le prie de transmettre ses excuses à l'Impératrice pour ne pas l'avoir remerciée de ses «félicitations" (pour sa réception à l'Académie ?). L'écrivain motive déjà son impossibilité d'aller à Rome à cause du manque de temps et d'un excès de travail. Le dernier "petit livre» dont il est question, non envoyée ${ }^{24}$ car hétérogène, avec des articles et des récits divers, pourrait être L'Exilée d'avril 1893, qui fait seulement 139 pages. En outre, selon son Journal, Loti, comme il l'annonce dans sa lettre, se trouve en effet à Paris le 1er février 1895, pour le mariage de Georges de Ferrière. Les jours suivants, jusqu'au 5 février, il rencontre Daudet, SullyPrudhomme, les princesses Bibesco et Sarah Bernhardt ${ }^{25}$ :

Monsieur,

Avec quelle confusion je retrouve aujourd'hui la lettre que vous aviez bien voulu m'écrire! Elle avait été noyée, par mégarde, dans le monceau de toutes celles, d'écritures inconnues, qui étaient restées en souffrance pendant mon voyage en Orient. Si mon secrétaire, improvisé à cette époque-là, avait seulement jeté les yeux sur les premiers mots : "L'Impératrice me charge etc. » il me l'aurait fait suivre là bas, avec une mention spéciale. La faute en est surtout à son étourderie. J'espère que vous voudrez bien me pardonner. Mais comment faire agréer mes humbles excuses à Sa Majesté? Aurez-vous la bonté d'essayer? Je vous en serai si reconnaissant, et je suis si désolé de n'avoir pas paru comprendre tout le prix des félicitations qu'Elle a daigné m'envoyer et dont je suis touché infiniment, serait-il possible d'en douter. Je vous en supplie, quand vous trouverez une occasion favorable, mettez à Ses pieds mes regrets si sincères, avec mes respects les plus profonds. Et, si un jour vous avez la bonté de me dire que vous l'avez fait, j'aurai l'esprit plus tranquille après.

Je n'ai pas osé envoyer à Sa Majesté mon dernier petit livre qui n'était qu'un assemblage de notes et d'articles publiés un peu partout. Quand j'aurai écrit une cuvre plus sérieuse, je vous demanderai la permission de la faire passer par vos mains, pour qu'elle arrive jusqu'à l'Impératrice si vous le jugez possible.

Helas [sic]! Aller vous voir à Rome, je le voudrais bien, si mon temps n'était toujours escompté, heure par heure, des mois à l'avance. Mais si vous êtes à Paris quand j'y viendrai en février, je serai bien heureux de me présenter chez vous. 
Veuillez agréer, je vous prie, monsieur, avec toutes mes excuses, mes sympathiques hommages

Pierre Loti

19 3) Lettre $n^{\circ} 1879$ sur papier avec monogramme et cartouche, datée "Préfecture 10 settembre » [1898 ?] : Loti est à terre, au port de Rochefort, du 15 avril 1898 au 1er mai $1899^{26}$. Il s'adresse, puisqu'il signe avec son deuxième vrai nom, à une amie, à laquelle il demande de le rejoindre et qu'il charge de présenter au comte peut-être la lettre d'excuses destinée à la princesse, $n^{\circ} 12361$ (infra $n^{\circ}$ 5), et celle pour le comte lui-même, $n$ - 1878 (infra n $\left.{ }^{\circ} 4\right)$ :

Préfecture 10 septbre

Voici la lettre pour être montrée à M. Primoli, si elle vous convient comme cela. Celle pour lui, je l'ai étourdiment laissée à la maison dans mon bureau. Vous la recevrez demain. Encore rien de vous au courrier de ce matin. Alors soyez bonne, dites moi par dépêche que vous viendrez.

Votre

Julien

4) La lettre au comte Primoli n ${ }^{\circ} 1878$ sur papier avec monogramme et cartouche, date du lendemain, le 11 septembre. Le refus de l'invitation à Rome, en raison de ses trop nombreux engagements et de son inexpérience à tenir des conférences, en particulier devant un public de dames de l'aristocratie, n'est pas définitif. L'indication dans la lettre du départ pour la Perse - le « grand voyage d'Orient » - confirmerait la date de septembre 1898. Dès décembre 1898, Loti commence à évoquer dans son Journal ses démarches et ses préparatifs en vue de son "grand voyage d'Orient $»^{27}$. La prévision du voyage revient au cours des premiers mois de 1899 ; le 6 janvier 1899 : « cet Orient où j'irai chercher un peu d'oubli ${ }^{28}$; le même mois, le $8:$ «Et je m'occupe au moment de ce départ si prochain pour l'Orient, de mes dispositions de mort, de mon testament $»^{29}$; le 27 : «[...] l'Orient dangereux où je m'enfoncerai bientôt... »" ; et le 5 février : "Est-ce que ce sera l'Orient délicieux et plein d'imprévu? $»^{31}$, jusqu'au 19 septembre, à la veille du grand départ, passée à Rochefort : «Et je regrette infinément d'avoir quitté Hendaye - tant cette grande maison de Rochefort me paraît vide et désolée $»^{32}$. C'est seulement le 19 novembre 1899 que Loti partira de Marseille pour l'Orient - l'Inde, la Perse... - dont il rentrera à la fin juin 1900 :

\section{Rochefort 11 septbre}

Monsieur,

Vous m'avez écrit une lettre bien charmante et vous l'avez fait passer par l'amie que je vénère et que j'aime le plus; aussi m'est-il extrêmement pénible de refuser ce que vous voulez bien me demander.

Mais j'y vois tant d'impossibilités, au milieu de ma vie en partie double ou triple, qui se consume dans un continuel surmenage! Comment pourrais-je aller à Rome cet hiver, quand mes moindres moments seront comptés, à la veille d'un grand départ pour la Perse et le Belouchistan [sic] ? Et puis, des conférences, je n'en ai jamais fait ; c'est bien en dehors de ma voie; j'y serais fort ennuyeux sans doute et vous ne perdrez rien à ne pas m'entendre.

Cependant je serais si désireux de répondre à votre invitation que je préfére ne pas vous envoyer encore un refus absolu. Voudrez-vous prendre toutes vos dispositions sans compter sur moi ; mais à la dernière heure, si je puis humainement venir, je viendrai, au moins assister à la fête, prouver ma bonne volonté par ma présence si je n'ai pas eu le temps de préparer la conférence demandée.

Veuillez, je vous prie, monsieur, me mettre aux pieds des dames romaines et agréer, avec mes excuses, l'hommage de lointaine sympathie,

Pierre Loti 
5) Lettre $n^{\circ} 12361$, sur papier avec monogramme et cartouche, à la princesse Mathilde, où Loti montre de nouveau qu'ils se connaissent bien et s'excuse d'avoir décliné l'invitation de son neveu à tenir la conférence à Rome :

\section{Princesse amie,}

J'ai tardé à répondre pour me donner le temps de réfléchir un peu. Il m'était si pénible de refuser, à une lettre si charmante et appuyée par vous - - Mais vous vous doutiez bien, n'estce pas, - me connaissant et sachant ma vie surmenée - qu'on me demandait là presque l'impossible. Voudrez vous être assez bonne pour transmettre la réponse ci jointe à $M$. Primoli et pour y ajouter quelques mots le priant de ne pas m'en vouloir, - ni lui, ni les dames romaines; j'en serais trop désolé. Ce que je lui dis est vrai : si je puis humainement aller à Rome et faire cette conférence, je la ferai.

Pardonnez-moi, vous aussi, princesse, et daignez agréer l'hommage de tout mon tendre respect.

$$
\text { P. Loti }
$$

Une dernière chose : si par hasard j'allais à Rome, je voudrais qu'il fût d'avance bien entendu qu'il ne sera jamais question de payer cette conférence; cela me désobligerait infiniment.

6) Le 2 août 1900, Loti part de Cherbourg pour la Chine et rentre en France le 7 avril 1902. Au Musée Napoléonien, l'enveloppe $n^{\circ} 5885$ adressée au « Comte Joseph Primoli a Roma, Italia ", contient une carte de visite, qui accompagnait peut-être un cadeau, avec le nom Pierre Loti imprimé au centre et, au-dessous, "Capitaine de Frégate»; dans la partie inférieure gauche « à bord du "Redoutable" » et dans la partie inférieure droite « Mers de Chine ». Au centre, sur une ligne écrite à la main : [Pierre Loti] « prie le comte Primoli de vouloir bien agréer l'expression de sa sympathie profonde. Mers de Chine, Déce 1901 ».

Le deuxième groupe de lettres sur papier simple, sans monogramme ni devise, se situe entre la fin de 1903 et le début de 1904, après l'invitation à dîner de la princesse Mathilde à l'écrivain le soir du 1er juin 1903, dernier événement mondain de la vieille dame, désormais âgée de plus de quatre-vingts ans.

La biographe et historienne Marguerite Castillon du Perron reconstruit dans un article ${ }^{33}$ cette soirée qu'elle situe au château de Saint-Gratien, comme s'il s'agissait d'une première rencontre. La princesse aurait reçu dans sa demeure d'été Loti et les d'Ocagne qui le lui avaient fait connaitre. Pendant la soirée, le souvenir d'Edmond de Goncourt, mort en 1896 chez Alphonse Daudet, fut évoqué : «Mathilde regrettait la publication du Journal qui, sous prétexte de pittoresque, contenait tant d'indiscrétions [...]. La Princesse n'était pas non plus bien contente de nombreux passages où son ami la peignait $»^{34}$. Au dire de la princesse, perpétuellement occupé de lui-même, irritable, nerveux, Edmond de Goncourt ne s'était remis de la mort de son frère que pour tomber entre les mains de la famille Daudet. La biographe et romancière raconte :

Loti admire cette très vieille dame [...] pas très grande, droite sur son canapé recouvert d'une perse à fleurs, ses cheveux sont ramenés en une natte au sommet de la tête, son profil est ferme, sa peau très blanche, étonnement lisse [...]. Mathilde l'observe aussi, petit, presque trop élégant dans son uniforme de capitaine de frégate. Elle l'interroge sur ses voyages, sur ses projets. ${ }^{35}$

Castillon du Perron remonte dans son article à quelques années plus tôt, au 10 octobre 1894, date d'une soirée où Maurice d'Ocagne ${ }^{36}$ entretient longuement la princesse et ses hôtes - Edmond de Goncourt faisait partie des invités - d'un écrivain qui, bien que fort jeune encore, vient d'entrer à l'Académie française. Ocagne fait le récit de l'étonnant dîner Louis XI que Loti avait offert le jeudi 12 avril 1888 à Rochefort : le thème de la soirée était l'âge des croisades. Loti met en scène des tables, des sièges, des objets usuels de l'époque et des plats préparés d'après des recettes anciennes. Les invitations étaient 
écrites en caractères gothiques. Chaque invité était travesti avec le plus grand souci d'exactitude et devait s'exprimer en vieux français. De véritables comédies avaient été montées : les acteurs jouaient des pèlerins, des mendiants, des jongleurs, des sorcières...

Edmond de Goncourt avait écrit dans son Journal à la date du mercredi 10 octobre 1894 : "Maurice d'Ocagne nous peint le côté amoureux de travestissement chez l'écrivain, dont la vie est un perpétuel carnaval, avec sa chambre bretonne où il s'habille en Breton, avec sa chambre turque où il s'habille en Turc, avec sa chambre japonaise où il s'habille en Japonais ». Ce passage paraît dans l'Écho de Paris du 28 mai 1896. Loti, froissé, écrit à Ocagne cette lettre qui, selon Castillon du Perron est inédite et a été communiquée par la famille Ocagne : «[...] où diable m'avez-vous vu déguisé en quelque chose, en dehors de la petite soirée à laquelle j'ai eu le plaisir de vous recevoir ? $\aleph^{37}$.

Loti envoie à Maurice d'Ocagne une seconde lettre, que Castillon du Perron présente comme inédite, mais que d'Ocagne avait déjà publiée en 1928 dans ses Souvenirs et causeries :

Nos lettres se sont croisées, et je ne vous en veux plus du tout après la vôtre. Je m'en excuse au contraire de ce mouvement de vivacité, mais si vous saviez la scie que ça est depuis des années pour moi, ce «perpétuel carnaval». Vous me feriez plaisir en disant à cette vieille portière de Goncourt qu'il nous a été désagréable à tous deux [...].38

Ocagne précise en note: «Je supprime, au reste, ici une épithète un peu trop vive pour être reproduite ", car il censure et remplace par des points de suspension l'expression « cette vieille portière ».

Dans sa biographie de la princesse Mathilde de 1988, Jean des Cars reprend avec quelques ajouts pittoresques l'anecdote de la visite du 1er juin 1903, en suggérant qu'il s'agit d'une première rencontre. La princesse reçoit à Saint-Gratien :

[...] un auteur à part dont lui avait parlé Ernest Renan, célèbre depuis 1886 pour son roman Pêcheur d'Islande [...] il lui parle des Japonaises qu'il juge des « insectes", de l'Inde, des mirages de la vieille Turquie où il va se rendre, des roses d'Ispahan. Le lieutenant de vaisseau préfère la chimère orientale à la convention européenne. Avec la princesse, il a un goût commun : l'art extrême oriental. Mais comme il a un genre bizarre... Elle remarque qu'il porte des talons hauts et qu'il est maquillé. Elle ne sait pas encore que Pierre Loti aura été son dernier invité. ${ }^{39}$

30 Les deux biographes indiquent la date exacte de la soirée, mais le lieu accrédité de la rencontre dans la résidence d'été de la princesse à Saint-Gratien est démenti par le Journal de Loti. Le dîner a indéniablement eu lieu à Paris, dans l'hôtel de la princesse, rue de Berri ${ }^{40}$ :

Samedi 30 mai

Arrivé hier au soir de Rochefort et installé ce matin à Paris, à l'hôtel Meurice.

[...]

Lundi 1er juin

À Paris, hôtel Meurice, balcon donnant sur le jardin des Tuileries.

Dîné chez la vieille princesse Mathilde, dans la stricte intimité, mais toujours au milieu

d'une grande magnificence impériale, avec partout des aigles et des abeilles.

À onze heures et jusqu'à minuit et demi, chez la princesse Alice, toujours dans l'angoisse pour son ami mourant.

Le 3 juillet 1903, la princesse tombe dans le petit escalier de son atelier à Saint-Gratien, où elle a pris comme d'habitude ses quartiers pour l'été, et se casse le col du fémur. Le comte Primoli prend dans un carnet des notes - en partie inédites ${ }^{41}$ - sur la longue maladie et 
l'agonie de sa tante. Le 1er octobre, la princesse rentre à Paris à l'hôtel Meurice. Au soir du 2 janvier 1904, elle s'éteint.

7) Lettre à la princesse Mathilde $n^{\circ}$ 12363, probablement de juillet-août 1903 ; l'écrivain a appris en retard l'accident, il remercie pour l'invitation du début juin et annonce une visite, avant son départ pour la Turquie, où il sera déjà en septembre 1903 :

Madame,

J'ai appris aujourd'hui seulement l'accident arrivé à Votre Altesse Impériale, et ma lettre sera presque un peu ridicule à présent que, Dieu merci, toute crainte est écartée; mais je suis l'homme qui n'ouvre jamais un journal et ignore tout ce qui se passe; j'espère que Votre Altesse voudra bien me pardonner.

Je pars dans quelques jours pour aller commander la station navale de Constantinople. Avant de m'en aller, j'irai m'inscrire rue de Berri; mais ce sera une façon si banale de reconnaître l'accueil reçu, que je demande la permission à Votre Altesse de lui dire ici le charmant souvenir que je garde de la soirée passée auprès d'Elle.

Je suis, avec un profond respect, Madame,

De Votre Altesse Impériale,

Le dévoué serviteur

Pierre Loti

8) Lettre au comte Primoli $n^{\circ}$ 1875, avec seulement la date du 21 août, probablement 1903, écrite à Hendaye. Loti se réjouit des nouvelles rassurantes sur la santé de la princesse, il demande au comte un portrait de sa tante et annonce son départ imminent pour l'Orient. Werther, le drame lyrique de Massenet cité dans la lettre, représenté au Châtelet le 16 janvier 1893, s'était imposé en France à l'Opéra Comique dans la mise en scène d'Albert Carré le soir du 24 avril 1903. Il fut peut-être l'occasion d'une rencontre mondaine entre Loti et le comte :

Hendaye 21 août

Monsieur,

Je suis tout heureux des bonnes nouvelles que vous voulez bien me donner de la Princesse Mathilde, et charmé du choix de l'intermédiaire, - car je n'ai point oublié nos correspondances anciennes, ni notre rencontre le soir de Werther. Voudrez-vous encore être assez bon pour transmettre à Son Altesse les vœux que je fais pour son complet rétablissement.

Il me reste à vous adresser une demande; si elle vous semble indiscrète, voudrez-vous tout simplement la considerer [sic] comme non avenue. Il y a longues années, depuis ma première jeunesse et le temps où j'étais parfaitement obscur, je savais déjà beaucoup de la Princesse, et me sentais attiré par cette personnalité si particulièrement vivante, intelligente et bonne. Je désirais donc conserver un portrait de Son Altesse ; mais, le mois dernier, j'en ai vainement cherché sous les arcades de la rue de Rivoli. S'il vous était possible de me faire obtenir la moindre petite photographie, vous n'imaginez pas combien vous me feriez plaisir; mais je reconnais que je suis très audacieux de demander cela.

Je repars dans une dizaine de jours pour mon cher Orient, que je vais retrouver, helas [sic]! bien bouleversé.

Veuillez agréer, je vous prie, monsieur, l'expression de mon plus sympathique souvenir, Pierre Loti

9) Lettre au comte Primoli n 1876 pour le remercier de l'envoi du portrait de la princesse. Loti, qui révèle ici indirectement sa fréquentation du salon de la princesse, rend la politesse en envoyant une photo de lui, conservée dans les archives de la Fondation Primoli ${ }^{42}$, et il lance un vague " au revoir à Rome » :

Cher monsieur,

Combien vous m'avez fait plaisir et combien je vous remercie! Le portrait que vous m'avez envoyé est la reproduction de celui que j'avais admiré dans le grand salon de son Altesse, et qui est si fidèle et si vivant. Je serai très heureux de vous envoyer le mien, puisque 
vous voulez bien le demander; je n'en possédais plus, mais j'en ai commandé chez mon photographe, et vous le recevrez bientôt.

Je vous dis au revoir à Rome, cher monsieur, et vous prie de vouloir bien agréer la nouvelle expression de mes sentiments très reconnaissants et sympathiques

Pierre Loti

comte Joseph Primoli, très sympathique souvenir $»$ :

Cher monsieur,

Merci de tout cœur pour le souvenir que je viens de recevoir et qui, je vous assure, m'est infiniment précieux. À moins que vous ne me donniez avis contraire, je voudrais écrire demain un mot de remerciement à Sa Majesté.

En hâte extrême, je vous prie de vouloir bien agréer, cher monsieur, l'expression de mes biens sympathiques sentiments,

Pierre Loti

11) Lettre ${ }^{43}$ à la princesse Mathilde $n^{\circ} 12362$, du 25 août [1903] où il la remercie pour avoir reçu la photo demandée, avec la signature de la princesse, et déclare qu'il fait voyager avec lui les objets qui lui sont chers, avant de les installer dans sa surprenante maisonmusée de Rochefort. Loti annonce son départ pour la Turquie comme commandant - il l'a été de septembre 1903 jusqu'à 1905 - de l'aviso-torpilleur Vautour :

Bakharetchea - Hendaye - 25 août

Madame,

À peine partie ma lettre au comte Primoli, j'ai été effrayé de l'indiscrétion de ma demande. Or, voici que je reçois ce matin le portrait que je désirais, et Votre Altesse a daigné le signer, ce qui est plus que je n'espérais. Combien je suis honoré et heureux de posséder ce précieux souvenir!

Les choses auxquelles je tiens, lorsqu'elles sont transportables, commencent toujours par faire une campagne avec moi, avant de prendre leur place définitive dans ma maison de Rochefort. Et la photographie que je viens de recevoir va d'abord m'accompagner pendant dix-huit mois sur le navire que je dois commander dans le Levant. Je ne sais comment en remercier Votre Altesse.

Je repars la semaine prochaine pour cet Istamboul, un des coins de la terre que j'ai le plus aimé.

Je suis, avec le plus profond respect, Madame,

De Votre Altesse Impériale,

Le reconnaissant et dévoué serviteur,

Pierre Loti

Lettre au comte Primoli $\mathrm{n}^{\circ} 1877$, de janvier 1904, de condoléance pour la perte de sa tante. Loti évoque sa tante Clarisse, Clarisse Texier, morte en 1890, à la mémoire de laquelle il a dédié Tante Claire nous quitte dans Le Livre de la pitié et de la mort de 1891, et qui apparait souvent dans son Journal associée - deux figures vêtues de noir - à sa mère :

"Vautour", Constantinople, 11 Janvier

Cher monsieur,

En rentrant avec mon navire à Constantinople, avant hier, j'ai appris la mort de la bonne et charmante Princesse. J'ai perdu, moi aussi, une vieille tante que j'adorais, et je puis si bien comprendre votre chagrin.

Comme il me devient précieux le portrait que vous avez eu la bonté de m'envoyer.

Veuillez agréer, je vous prie, cher monsieur, l'expression de ma sympathie profonde,

Pierre Loti

13) Carte postale au comte Primoli $n^{\circ} 1882$. La Salle Chinoise ${ }^{44}$, créée en 1902 , avait été inaugurée en mai 1903 avec une fête somptueuse :

ROCHEFORT. Maison de Pierre Loti. La Salle Chinoise 
Merci, cher Monsieur, de ne pas m'oublier. Veuillez agréer, je vous prie, mes meilleurs vœux et mes bien sympathiques souvenirs. romaine, mais peu enclin à visiter Rome, capitale de l'Antiquité classique et de la papauté, peut-être dépourvue de mystère et d'un charme exotique à découvrir, et pourtant hantée par le fantôme de l'écrivain que quelques témoins affirment même y avoir rencontré.

\section{NOTES}

1. Du journal autobiographique du comte (1851-1927), il n'a été publié jusqu'à présent que : G. Primoli, Mémoires, 1851-1871, texte intégral inédit, M. Colesanti - V. Petitto (éd.), Rome, Edizioni di Storia e letteratura, «Quaderni di Cultura Francese », 2012, p. XXXVI-584. Voir l'introduction de M. Colesanti pour la vie et la physionomie intellectuelle du Comte, et les indications bibliographiques sur ses écrits et sur les études le concernant. Cette citation se trouve à la p. 511 . J'exprime toute ma reconnaissance au Président de la Fondation, et je remercie sa collaboratrice si compétente, Valeria Petitto, pour avoir mis à ma disposition les livres et les lettres de Pierre Loti, ainsi que la page du manuscrit du comte, et pour m'avoir concrètement aidée dans mes recherches.

2. M. Borgese dans la biographie La Contessa Lara, Milan, Garzanti, 1930, pseudonyme d'Evelina Cattermole, liée au jeune poète et journaliste de la Cronaca Bizantina, G. A. Cesareo, et tuée à Rome en 1896, affirme aux pages 153-156 que chez les deux poètes se réunissait un petit nombre d'amis et d'écrivains, y compris étrangers, comme P. de Nolhac et Loti, chaque fois qu'il venait à Rome. La comtesse aurait écrit dans ses Cronache Femminili parues dans la Tribuna Illustrata de 1892 quelques vers inspirés par Madame Chrysanthème. À son enterrement, il y aurait eu une couronne de fleurs envoyée par Loti (La Contessa Lara, cit., p. 253). G. Natali, Profili di maestri e amici, Rome, Edizioni di Storia e Letteratura, 1965, résume à la p. 128 : un groupe élu d'amis fréquente le salon littéraire de la comtesse, dont P. de Nolhac et Loti. Affirmation reprise par B. Schisa dans Dopo ogni abbandono. Il romanzo della Contessa Lara, amore e morte nella Roma dell'Ottocento, Milan, Garzanti, 2009, p. 163 et 39 : à Rome, autour de la table en noyer de la salle à manger de la comtesse, étaient assis ses amis hommes de lettres, qui l'avaient toujours soutenue, L. Capuana, G. D'Annunzio et Loti.

3. A. Bertuccioli, Pierre Loti. Vita e opera, Milan, Fratelli Treves Editori, 1926, p. X. Ce petit livre aborde dans ses différents chapitres: I. La vie et les écrits biographiques; II. Les romans d'amour ; III. La Bretagne - Le pays basque ; IV. Par les amples routes du monde ; V. Luttes d'âmes - Batailles de peuples. Les notes et la bibliographie révèlent une information et une bonne connaissance des études françaises de H. Bordeaux, P. Bourget, F. Brunetière, R. Doumic, E. Faguet, P. Faure, A. France, R. Lalou, Ch. Le Goffic, J. Lemaître, H. de Régnier, N. Serban. (« Loti n'avait jamais nourri, Dieu sait pourquoi ?... (préjugés surannés?... Mauvaise évaluation de certaines conditions politiques ?...) une grande sympathie pour la jeune Italie », c'est moi qui traduis).

4. P. Loti, « Impressions d'Italie », dans Id., L'Horreur Allemande, 1918 : Loti rencontre le roi VictorEmmanuel et une Eleonora Duse désormais chenue, amie chère et correspondante du comte Primoli qui la photographie à Venise en 1894 (Archives de la Fondation) ; Id., Impressioni d'Italia, préface de Louis Barthou, traduction et introduction d'Americo Bertuccioli, Lanciano, R. Carabba 
Editore, «L'Italia negli scrittori stranieri », 1931. Bertuccioli réintroduit dans sa traduction une page censurée par l'Illustration française où Loti décrivait les canons monstrueux cachés dans les lagunes pour défendre Venise, «en tenue de guerre». Barthou, avec qui Bertuccioli était en correspondance, témoigne dans sa préface de l'admiration de Loti pour l'armée italienne et affirme que ce reportage de guerre allait susciter de nouveaux sentiments en Italie en faveur de Loti, p. 26 : comme exemple, il cite l'article de G. Cartella Gelardi, « Onoriamo Pierre Loti », dans Il pensiero, Bergame, 15 octobre 1930. Le passage censuré et retrouvé par le fils de Loti est à la p. 57. Bertuccioli avait publié "Une page inédite de Pierre Loti sur l'Italie», dans Revue des Études Italiennes, 1931, p. 108-111; et P. de Nolhac, «Pierre Loti et les Italiens », dans Le Figaro, 5 mars 1931, p. 5.

5. "J'oserais dire que même la guerre italienne lui importait peu » : U. Ojetti, Cose viste, avec une prose de G. D'Annunzio, 1921-1927, t. I, Florence, Sansoni, 1951, p. 291-294. Dans les Archives de la Fondation, une photo du comte montre au palais Primoli en 1895 un groupe de gens de lettres, dont Matilde Serao, F. Martini, C. Pascarella et Ojetti. En outre, pour la fortune limitée de Loti en Italie : un court profil, Vicenda di Loti, se trouve dans C. Cordié, Saggi e studi di letteratura francese, Padova, Cedam, 1957, p. 145-151; B. et G. Melchiori, «Le suggestioni di Loti », dans Il gusto di Henri James, Turin, Einaudi, 1974, p. 237-266 ; A. M. Scaiola, « Pierre Loti », dans M. Colesanti-L. de Nardis (dir.), I contemporanei francesi, t. I, Rome, Lucarini, 1976, p. 145-153 ; F. Fiorentino, « Sogno esotico e racconto onirico, "Aziyadé" ", dans Rivista di letterature moderne e comparate, XXXVI, 1983, p. 25-48 ; Id., " Récit exotique et récit onirique. “Azyadé” de Pierre Loti », dans M. Ipotési (dir.), Pierre Loti et l'exotisme fin de siècle, Tarante, Lisi, 2007, p. 13-36 : contributions de M.G. Porcelli, A. Di Benedetto, M. Ipotési... M. Colesanti dans La letteratura francese dal Romanticismo al Simbolismo, Milan, Rizzoli BUR, 1992, lui consacre les p. 396-398.

6. Jusqu'en 1905, l'éditeur Lévy ne prend pas de trait d'union. Cf. P. Loti, Journal, vol. III : 1887-1895, A. Quella-Villéger et B. Vercier (éd.), Paris, Les Indes savantes, 2012, p. 808.

7. J'utilise les italiques pour des citations de passages inédits.

8. H. de Régnier, Escales en Méditerranée, Paris, Ernest Flammarion Éditeur, 1906. Dans Id., De mon temps, Paris, Mercure de France, 1933, Régnier trace un portrait évocateur du comte, voyageur et photographe, en racontant la croisière de deux mois en 1906 sur le Nirvana de la Comtesse de Béhague.

9. Il s'agit de la princesse Maria Carolina Rospigliosi Pallavicini, belle-sœur de Teresa Boncompagni, grande amie du comte. Deux photos du comte datées du 24 octobre 1896, conservées dans les Archives de la Fondation, montrent la princesse aux noces du prince de Naples Victor-Emmanuel avec Hélène du Montenegro.

10. Sur le Comité, voir : M. Spaziani, «"Carità e lavoro". Roma 1897 », dans Studi Romani, 4-5, septembre-octobre 1956, p. 570-577. Pour cet auteur, la plupart des photos qui accompagnent le fascicule - Roma, Forzani e Cie -, dont la Fondation Primoli possède un exemplaire, sont du comte, qui signe une nouvelle, La route de Rome, illustrée de vues de la ville. De M. Spaziani, cf. aussi Con Gégé Primoli nella Roma bizantina, Rome, Edizioni di Storia e Letteratura, 1962. Giuseppe Primoli était aussi appelé Joseph, ou surnommé plus familièrement Gégé.

11. J. P. Lion cite le quatrain de Mallarmé, publié ensuite dans Vers de circonstance, chap. Fêtes et anniversaires, $\mathrm{n}^{\circ} \mathrm{XVI}$, dans son essai «Le comte Joseph Primoli et les lettres françaises ", tiré à part de la revue Histoire Littéraire, 42, 2010.

12. P. Loti, Journal, vol. IV : 1896-1902, A. Quella-Villéger et B. Vercier (éd.), Paris, Les Indes savantes, 2016, p. 84-88. Pour les datations des lettres, nous nous référons aux chronologies précises établies par les éditeurs.

13. Ibid., p. 528.

14. Cf. R. Lefêvre, "Un collaborateur de Loti : Jousselin, dit Plumkett », dans Revue d'histoire littéraire de la France, 44e année, 3, 1937, p. 420-428. Sur les rapports de Loti avec le monde de 
l'édition : J.-Y. Mollier, L'Argent et les lettres : histoire du capitalisme d'édition, 1880-1920, Paris, Fayard, 1988.

15. Inv. MN _9273/I. Les journaux de Giulia, conservés au Musée Napoléonien, contiennent des évocations des livres de Loti. Elle écrit le 10 avril 1898 : "Je reviens de la messe à S[ain]t Louis des Français où j'ai bien prié pour Laetitia, l'amour de ma vie; j'ai déjeuné avec Lucien, Valérie et le commandant Jousselin, qui nous a parlé de la Chine et du Japon, ainsi que de l'écrivain Pierre Loti, avec le quel il est très lié ", dans Cahier n 56, MN_10401, p. 59-60.

16. Je remercie encore pour son aimable attention Alain Quella-Villéger qui m'a signalé ces lettres.

17. P. Loti, Journal, 1896-1902, cit., p. 115.

18. Dans son volume Gli amici della principessa Matilde. Lettres inédites de Mérimée, Sainte-Beuve, Gautier, Flaubert, Renan, Taine, Goncourt, Maupassant, Rome, Edizioni di Storia e Letteratura, "Quaderni di Cultura Francese », 2, 1960, M. Spaziani ne cite pas les lettres de Loti. Marguerite Castillon du Perron est l'auteure de la biographie, La Princesse Mathilde, Paris, Librairie Académique Perrin, 1953, bien documentée également en raison de la consultation du Fonds Grandjean et du Fonds Primoli en 1948, dont elle cite des documents et des lettres inédites, mais pas celles de Loti.

19. Son séjour à Venise à l'hôtel Danieli est évoqué par Loti dans L'Exilée de 1893. Sur Élisabeth de Roumanie, Loti avait écrit Carmen Sylva (1890). Cf. Pierre Loti et Carmen Sylva. Autour de l'Exilée, Sinala, Bucarest, Venise, textes croisés, réunis et présentés par A. Quella-Villéger, MignalouxBeauvoir, Le Carrelet Éditions, 2016. Le Journal, vol. III : 1887-1895, cit., p. 381, décrit à partir du 14 août 1891 le séjour à Venise : « Lumière cendrée, des extrêmes matins d'été, brume lumineuse, couleur gris de lin, d'avant le soleil levé ». Dans Impressions d'Italie, Loti rappelle avec mélancolie les journées passées une trentaine d'années plus tôt à Venise avec la reine Élisabeth, leurs lentes promenades nocturnes en gondole, et il rend hommage à la souveraine.

20. P. Loti, Journal, vol. III : 1887-1895, cit., p. 439.

21. Ibid., p. 520.

22. Titre aussi d'un poème de J. Moréas, Poésies (1886-1896), Paris, Bibliothèque artistique \& littéraire, 1898, p. 81. A. Quella Villéger, «Fixer dans la pierre le temps nomade. La Maison de Pierre Loti à Rochefort Palais des voyages devenu objet touristique", Actes de la "Journée Architectures ", Saint Malo (Assoc. "Pour ainsi dire"), mars 2009, p. 49-81, qui reprend l'essentiel du texte Chez Pierre Loti - Une maison d'écrivain-voyageur, Paris, SCÉRÉN-CRDP PoitouCharentes, 2008 [DVD]. « Mais la phrase entre toutes, enrubannée et imprimée sur son papier à lettre est la devise même de Loti, “Mon mal j'enchante”. Elle n'est pas originale ; Don Quichotte rapporte lui-même avoir entendu dire que "qui chante son mal l'enchante" et elle a été reprise, avec quelques variantes, par des poètes de la Pléiade ou par Aubanel. Elle mêle l'amour à la mort, le plaisir à la douleur, mais, attention, la devise n'est pas, comme on l'écrit parfois, "mon mal m'enchante", ce qui ressortirait du pur masochisme!». Id., Pierre Loti. Le Pèlerin de la Planète, Anglet, Auberon, 2005 ; Id., Chez Pierre Loti. Une maison d'écrivain-voyageur, Paris, SCÉRÉN-CRDP Poitou-Charentes, 2008 [DVD].

23. P. Loti, Journal, vol. III : 1887-1895, cit., p. 739.

24. Loti avait l'habitude d'envoyer ses livres à l'impératrice, puisque dans cette lettre autographe en vente le 10 décembre 2015 chez PIASA, 118, rue du Faubourg Saint-Honoré à Paris, avec un exemplaire de La mort de Philae, (1908), Eugénie le remercie du livre qu'elle a reçu. La lettre est datée du 19 février 1909, et signée : «Je vous remercie, monsieur, de m'avoir envoyé votre livre sur l'Égypte, à l'intérêt que je porte à ce beau pays auquel tant de souvenirs nous rattachent, se joint l'attrait que vous savez donner à tout ce que vous écrivez, et si j'y ai trouvé des regrets pour une époque dont la civilisation moderne transforme la beauté et viole le mystère, j'ai eu le plaisir de les voir exprimés dans une belle langue. Croyez, monsieur, à mes meilleurs sentiments. Eugénie ». Dans l'exposition organisée pour le centenaire de la naissance de Loti, Paris, 
Bibliothèque Nationale, 1950, figurent des lettres et une photographie dédicacée de l'impératrice à l'écrivain.

25. P. Loti, Journal, vol. III : 1887-1895, cit., p. 751-752.

26. Id., Journal, 1896-1902, cit., p. 692.

27. Ibid., p. 237.

28. Ibid., p. 243.

29. Ibid., 244.

30. Ibid., 247.

31. Ibid., 248.

32. Ibid., 301.

33. M. Castillon du Perron, « Une visite de Pierre Loti à la Princesse Mathilde », dans Les Cahiers de l'Ouest, 13, juillet 1954, p. 41-44.

34. Ibid., p. 43.

35. Ibidem.

36. Le mathématicien Maurice d'Ocagne est l'auteur des dernières années de la Princesse Mathilde, Paris, La Revue hebdomadaire, 1925. La Bibliothèque Primoli en possède un exemplaire avec dédicace : «Au Comte Primoli, avec mon sympathique souvenir. M. d'Ocagne ». Dans Souvenirs et causeries, Paris, Plon, 1928, Ocagne consacre le premier chapitre (p. 1-72) à la princesse. Aux pages 105-132, "Souvenirs familiers de P. Loti ", il évoque sa première rencontre en mai 1885 à Rochefort avec l'écrivain, que lui a présenté Mme Juliette Adam qui logeait dans la villa de Loti, aménagée selon le style des pays où il avait voyagé, et illustrée en détails par Ocagne. Aux pages 119-132, il décrit longuement l'étonnant festin époque Louis XI du 12 avril 1888, qu'Ocagne considère comme une véritable reconstitution historique, conforme aux données de la plus rigoureuse documentation, et il raconte le désagréable incident de la soirée du 10 octobre 1894 à la table de la princesse avec Edmond de Goncourt. Cf. A. Quella-Villéger, "Une fête médiévale chez Loti à Rochefort le 12 avril 1888 ", dans S. Abiker, A. Besson, F. Plet-Nicolas (dir.), Le Moyen Âge en jeu, dans Eidôlon, 86, décembre 2009, p. 263-275 [DVD-rom]. La veille de la fête, Loti écrit dans son Journal, vol. III, 1887-1895, cit., p. 163 : «J'attends les nuits avec une impatiente ivresse ".

37. M. Castillon du Perron, «Une visite de Pierre Loti à la Princesse Mathilde », cit., p. 43.

38. M. d'Ocagne, Souvenirs et causeries, cit., p. 129.

39. J. des Cars, La Princesse Mathilde. L'amour, la gloire et les arts, Paris, Perrin, 1988, p. 488.

40. Je dois encore une fois cette rectification et le passage du Journal de la fin mai 1903 à A. Quella-Villéger, éditeur avec B. Vercier de l'édition du Journal de Loti : le volume IV (1896-1902) est sorti début 2016, mais l'année 1903 sera dans le volume V. L'ami mourant de la princesse Alice de Monaco est le comédien britannique Isidore de Lara.

Seul Jérôme Picon, dans sa biographie Mathilde, princesse Bonaparte, Paris, Flammarion, 2005, p. 374 sq., indique qu'au tout début du mois [juin 1903], Mathilde avait encore reçu chez elle l'exotique marin, mais comme s'il était «nouveau rue de Berri ». Loti notait dans son Journal qu'il avait « dîné chez la vieille princesse Mathilde, dans la stricte intimité, mais toujours au milieu d'une magnificence impériale, avec partout des aigles et des abeilles ", passage cité par J. Picon et tiré de P. Loti, Cette éternelle nostalgie. Journal intime, 1878-1911, B. Vercier, G. Dugas et A. QuellaVilléger (éd.), Paris, La Table Ronde, 1997, p. 479.

41. J. Picon a consulté le manuscrit LIII, de la Fondazione Primoli, et inséré dans sa biographie le résumé des notes du comte dans un calepin sur l'évolution de la maladie de sa tante, son retour à Paris et ses derniers jours de souffrance. L'Illustration avait publié le 28 novembre 1903 en première page, pour rendre hommage à la princesse malade, un portrait de profil, peint en 1894 (Musée national des Châteaux de Versailles et de Trianon) par Henri-Lucien Doucet, où elle est représentée en train de travailler à une étude de fleurs, dans son atelier de Saint-Gratien. 
42. Photographie Delphin. E. Montastier. Rochefort S/Mer, en grand uniforme de Capitaine de frégate, avec médailles.

43. La lettre est datée de Bakharetchea, nom donné le dimanche 6 août 1899 à la maison d'Hendaye, qui veut dire : la maison du solitaire ou la maison solitaire. Cf. P. Loti, Journal, vol. IV : 1896-1902, cit., p. 294 et note 58.

44. Ibid., p. 683 : même le 25 décembre 1902, Loti suit les travaux des ouvriers dans la salle chinoise («c'est mon amusement de ce triste jour »).

\section{RÉSUMÉS}

Les documents conservés à la Fondation Primoli de Rome concernant Pierre Loti, qui a eu peu de contacts avec l'Italie où sa reception a été incertaine, permettent d'ajouter un certain nombre d'éléments peu connus ou inédits à sa biographie. En particulier, ses lettres autographes et inédites à la princesse Mathilde et au comte Primoli, qu'il eut l'occasion de fréquenter en France, confirment ses relations significatives avec les descendants italiens de Napoléon Bonaparte, tout en levant un doute quant à une visite présumée de l'écrivain-voyageur à Rome, plusieurs fois évoquée dans cette correspondance.

\section{INDEX}

Mots-clés : Loti (Pierre), Primoli (Joseph), Bonaparte (Mathilde), lettres inédites 